\title{
Algoritmos para Aumentar o Tempo de Vida de Redes de Sensores Sem Fio Utilizando Inovação
}

\author{
Felipe da Rocha Henriques, Lisandro Lovisolo e Marcelo Gonçalves Rubinstein
}

\begin{abstract}
Resumo-Redes de Sensores Sem Fio (RSSFs) são comumente utilizadas para realizar medidas em regiões que se deseja monitorar e enviá-las a uma estação base, ou sorvedouro. Um aspecto importante sobre RSSFs é a economia de energia pelos nós, o que acarreta em um aumento no seu Tempo de Vida. Este trabalho propõe dois algoritmos, um baseado na fonte e o outro no destino. Os algoritmos têm por objetivo fazer com que os nós economizem energia, através da diminuição da quantidade de transmissões pelos nós e do chaveamento destes para um estado de inatividade, entre transmissões. Para isto é utilizado o conceito de Taxa de Inovação. Os resultados mostram um aumento de até 194\% no Tempo de Vida da rede, com uma redução significativa na quantidade de transmissões dos nós sensores, em relação à situação onde não se utiliza nenhum gerenciamento de energia.
\end{abstract}

Palavras-Chave-Redes de Sensores Sem Fio, energia, taxa de inovação, inativo.

Abstract-Wireless Sensor Networks (WSNs) are generally used to sense regions and to send the sampled measurements to a base station, or a sink. One important aspect regarding WSN is to save energy at the sensor nodes, which may increase it's lifetime. This work proposes two algorithms, one that is source-based and another that is sink-based. These algorithms aim to save energy by decreasing the amount of transmissions and by making nodes switch to the inactivity state, between transmissions. To do this, the Innovation Rate concept is employed. Results show an increase up to $194 \%$ of the network lifetime, with a significant decrease in the amount of transmissions by sensor nodes, comparing to a network without any kind of node energy management strategy.

Keywords-Wireless Sensor Networks, energy, rate of innovation, inactive.

\section{INTRODUÇÃO}

Redes de Sensores Sem Fio (RSSFs) são comumente utilizadas para o monitoramento de regiões, a coleta e o repasse de dados [1]. Tais redes devem possuir algumas características importantes, como autoconfiguração (devido ao fato de poderem estar situadas em ambientes de difícil acesso, problemas de interferências e perda de nós) e restrições de energia, já que cada nó possui uma bateria e a troca desta na maioria das vezes é inviável [2].

Os sensores de uma RSSF possuem quatro unidades principais: unidade de processamento, unidade de comunicação, unidade de sensoriamento e unidade de energia. A última é composta geralmente por uma bateria, que atua como fonte de energia em cada componente dos nós [3].

*Felipe da Rocha Henriques, Lisandro Lovisolo e Marcelo Gonçalves Rubinstein, Faculdade de Engenharia, Programa de Pós-graduação em Engenharia Eletrônica (PEL), Universidade do Estado do Rio de Janeiro, Rio de Janeiro, *Centro Federal de Educação Tecnológica Celso Suckow da Foseca - Unidade Petrópolis, Rio de Janeiro, Brasil. E-mails: *henriquesfelipe@yahoo.com.br, lisandro@uerj.br, rubi@uerj.br
Uma das questões importantes a respeito das RSSFs é a energia gasta pelos nós sensores, que está diretamente relacionada com o conceito de Tempo de Vida da rede. Este pode ser entendido, em algumas situações, como o tempo em que o primeiro nó sensor morre, ou seja, quando sua bateria acaba [1]. Portanto, mecanismos que permitam economizar a energia dos nós sensores e, com isso, aumentar o Tempo de Vida da rede, devem ser utilizados para aumentar a autonomia da mesma. Muitos trabalhos propõem métodos para economizar a energia dos nós da rede, de modo a prolongar seu Tempo de Vida. Em [4], por exemplo, é realizado um controle de potência de transmissão. Um outro exemplo utiliza aspectos do roteamento de pacotes para economizar energia e, para isso, propõe uma modificação no protocolo de roteamento Direct Diffusion [5] [6].

Para alguns a tarefa mais custosa, em termos energéticos, de uma RSSF é a comunicação (transmissão e recepção), sendo seu consumo de energia muito maior do que aquele gasto no processamento de dados [3] [7]. Logo, é interessante utilizar métodos que minimizem a quantidade de transmissões, de modo a economizar energia. Pode-se verificar um exemplo desta estratégia em [8], que utiliza estimação de campo para diminuir a quantidade de amostras enviadas ao sorvedouro.

Da mesma forma, de modo a diminuir o gasto energético, em [9] utiliza-se a correlação espaço-temporal das amostras medidas pelos nós, com o intuito de reduzir a quantidade de transmissões. Além disso, nem todos os nós precisam estar ativos simultaneamente. Estes podem passar para um estado de inatividade (ou economia de energia) [4] e, com isso, prolongar o Tempo de Vida da rede. Outro mecanismo utilizado para o mesmo fim é a agregação de dados, método que explora a redundância dos dados coletados, de modo a economizar energia dos nós sensores [10].

Um conceito que pode ser explorado, com o intuito de minimizar o número de transmissões e, portanto, economizar energia é a Taxa de Inovação [11]. Os nós apenas transmitiriam medidas que apresentassem uma determinada quantidade de Inovação, ou um determinado nível de variação entre amostras do sinal medido.

Este trabalho propõe dois algoritmos para economia de energia dos nós sensores, um baseado na fonte e outro no destino. Esses algoritmos utilizam o conceito de Taxa de Inovação, de modo a gerenciar a necessidade de comunicação e, com isso, economizar energia. Além disso, a partir dessa taxa, explora-se o estado de inatividade dos nós sensores, entre transmissões, para minimizar o custo energético quando os nós não estão realizando comunicação. Os tempos de inatividade são inferidos através da Taxa de Inovação, ou seja, quanto 
menor for a taxa com que os nós realizam transmissões de medidas com Inovação, maior será o tempo em que os nós permanecerão Inativos e, portanto, economizando energia. $\mathrm{O}$ conceito de Taxa de Inovação pode ser visto, por exemplo, em [12], onde utiliza-se a Inovação como métrica para seleção de rotas em uma RSSF.

O problema descrito neste trabalho diz respeito à utilização de uma RSSF para realizar medidas em uma determinada região, o que será representado por uma superfície bidimensional variante no tempo. As medidas são transmitidas por cada nó sensor para um nó sorvedouro e os algoritmos para economia de energia são empregados de modo a aumentar a autonomia da rede, através do aumento do seu Tempo de Vida. Além da análise do Tempo de Vida da rede, deve-se levar em conta o erro na reconstrução das medidas. Quando os nós estão Inativos, eles não realizam medidas e, neste intervalo de tempo, o processo monitorado pode variar, levando a um erro na sua reconstrução. Portanto, deve existir um compromisso entre o aumento no Tempo de Vida da rede e o erro na reconstrução do processo.

A organização do trabalho é a seguinte: na Seção II serão discutidos os algoritmos propostos. A Seção III apresenta o modelo de energia utilizado no trabalho. A Seção IV trata de aspectos utilizados nas simulações, como o ambiente de simulação e seus parâmetros principais. A Seção V apresenta os resultados obtidos e, por fim, na Seção VI, são apresentadas as conclusões e os possíveis trabalhos futuros.

\section{Algoritmos para Economia De Energia}

\section{A. Algoritmo 1: Taxa de Inovação na Fonte}

Neste algoritmo será utilizado o conceito de Inovação na fonte, ou seja, em cada nó sensor. Inicialmente, cada sensor realiza a primeira medida e transmite a amostra para o destino. Chamaremos as medidas de $x_{i}(j)$, onde $i$ representa o sensor e $j$, a ordem sequencial das medidas. Então, a partir da segunda medida de cada sensor, esta será comparada com a anterior. $\mathrm{O}$ sensor então, irá transmitir apenas as amostras para as quais a variação percentual entre a amostra atual e a anteriormente transmitida seja maior do que um limiar pré-fixado. Assim, o sensor $i$ apenas transmite uma nova amostra $j+n$, caso

$$
\frac{\left|x_{i}(j+n)-x_{i}(j)\right|}{x_{i}(j+n)}>\text { limiar. }
$$

onde $x_{i}(j)$ é a última amostra transmitida pelo sensor.

Logo, apenas amostras com um determinado nível de Inovação serão transmitidas. Desta forma, espera-se reduzir a quantidade de transmissões de cada sensor e, com isso, aumentar o Tempo de Vida da rede.

Além disso, cada sensor, de forma autônoma, pode decidir passar para o estado Inativo após transmissões inovadoras, de modo a economizar energia. Uma estratégia em que os nós transitam para o estado Inativo após as transmissões é abordada em [13].

Se $\Delta \mathrm{t}$ for definido como o intervalo de tempo entre duas transmissões (com Inovação), o nó entrará no estado Inativo pelos próximos $\Delta \mathrm{t} / 2$ segundos. Isto para que, caso ocorra algum evento com variações mais bruscas, por exemplo uma explosão, no caso do monitoramento de temperatura, os nós possam voltar ao estado Ativo a tempo, de modo a não perder estas informações. Este tempo de inatividade não é fixo, e pode variar com $\Delta$ t. Após o tempo de inatividade, o nó retorna ao estado Ativo, mede novamente o processo e compara esta medida com a anterior (com Inovação). Este processo é realizado até que a energia do nó $s_{i}$ acabe.

Nesta abordagem, baseada na fonte, a partir do processamento local das amostras medidas, os nós são autônomos para decidir por transmitir ou não, passar para o modo Inativo e por quanto tempo permanecer neste modo.

A seguir, pode-se ver, para cada nó $s_{i}$, os passos do algoritmo baseado na fonte.

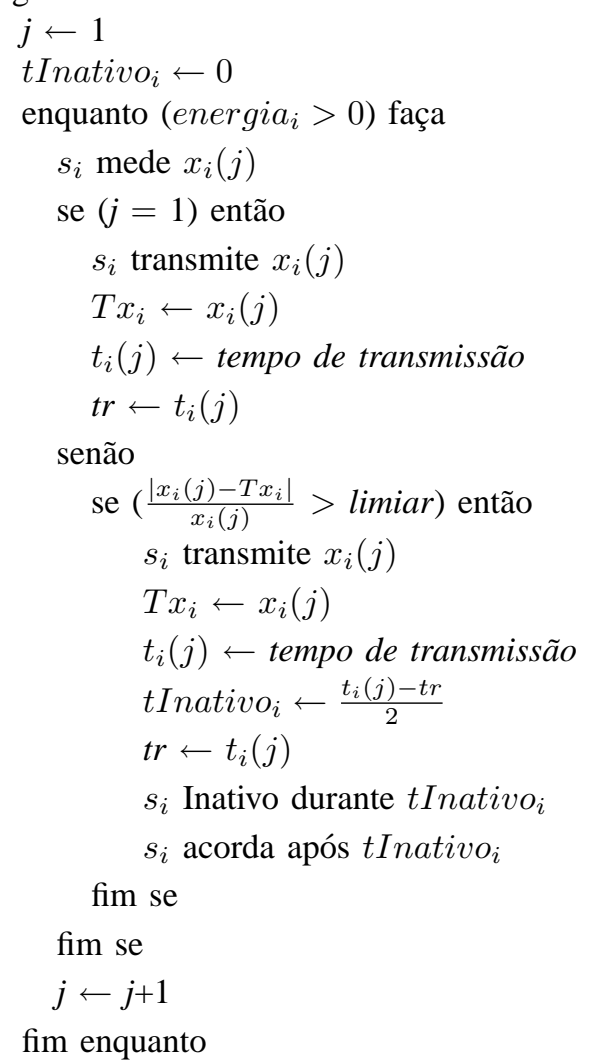

Deve-se observar que não se sabe, inicialmente, o tempo de inatividade de cada nó. Portanto, no começo do algoritmo, este é definido como tnativo $_{i} \leftarrow 0$. Logo, deve-se realizar medidas até encontrar este valor, a partir da diferença entre os instantes de transmissão de medidas com Inovação.

\section{B. Algoritmo 2: Taxa de Inovação no Sorvedouro}

Nesta abordagem, os nós sensores não precisam realizar nenhum processamento, tão pouco tomar decisões. Eles simplesmente medem as amostras e as transmitem para o sorvedouro. Este último é o responsável pela tomada de decisões. Para cada sensor, o sorvedouro, a partir da segunda amostra recebida, calcula a variação percentual, conforme a Equação 1. Seja $y_{i}(j)$ o primeiro valor recebido pelo sorvedouro, ou seja, a amostra $j$ do sensor $i$. A partir da próxima amostra recebida, o sorvedouro calcula a variação percentual entre a amostra atual $\left(y_{i}(j+n)\right)$ e a primeira. Caso esta variação seja maior do que um limiar pré-determinado, ou seja, Inovação, o sorvedouro calcula o intervalo de tempo $\Delta t$ entre as respectivas 
recepções e manda que o respectivo nó transmissor durma durante metade deste intervalo, portanto, $\Delta \mathrm{t} / 2$. Após este tempo de inatividade, que pode variar com $\Delta \mathrm{t}$, o nó sensor em questão volta ao estado Ativo e continua a transmitir amostras medidas para o sorvedouro. O sorvedouro realiza este processo de chaveamento de estados (Ativo/Inativo) dos sensores até que a energia do primeiro nó sensor $\left(s_{i}\right)$ acabe.

Observa-se que, neste algoritmo, todo o gerenciamento é realizado pelo sorvedouro, diferentemente da abordagem com Inovação na fonte, onde os sensores processam e tomam as decisões. Logo, o atraso na comunicação entre sensores e sorvedouro pode influenciar nos tempos de inatividade calculados. Porém, tal aspecto não foi considerado, já que todos os nós sensores estão dentro do raio de alcance do sorvedouro e a área monitorada não é muito grande.

A seguir é apresentado o algoritmo, para o sorvedouro, chamado de $s_{r}$.

$$
\begin{aligned}
& j \leftarrow 1 \\
& \text { tInativo }_{i} \leftarrow 0 \\
& \text { enquanto (energia } a_{i}>0 \text { ) faça } \\
& s_{r} \text { recebe } y_{i}(j) \\
& \text { se }(j=1) \text { então } \\
& R y_{i} \leftarrow y_{i}(j) \\
& t_{i}(j) \leftarrow \text { tempo de recepção } \\
& \text { tr } \leftarrow t_{i}(j) \\
& \text { senão } \\
& t_{i}(j) \leftarrow \text { tempo de recepção } \\
& \text { se }\left(\frac{\left|y_{i}(j)-R y_{i}\right|}{y_{i}(j)}>\text { limiar }\right) \text { então } \\
& \text { tInativo }_{i} \leftarrow \frac{t_{i}(j)-t r}{2} \\
& \text { tr } \leftarrow t_{i}(j) \\
& R y_{i} \leftarrow y_{i}(j) \\
& s_{i} \text { Inativo durante } \text { tnativo }_{i} \\
& s_{i} \text { acorda após } \text { tnativo }_{i} \\
& j \leftarrow j+1
\end{aligned}
$$

fim enquanto

Neste algoritmo, inicialmente, o tempo de inatividade é definido como tnativo $_{i} \leftarrow 0$, pelos mesmos motivos apresentados no algoritmo 1 . Neste caso, o sorvedouro deve receber amostras até encontrar este valor, para cada nó sensor.

\section{MOdELo DE ENERGIA}

O modelo de energia define os consumos de energia no decorrer das simulações. Em [14], são apresentados dois modelos de energia para redes ad hoc, um por pacotes e um por estados. O primeiro é função do tamanho do pacote, e o segundo leva em conta o tempo de permanência do nó em cada estado (Transmissão, Recepção, Dormência e Ocioso).

O modelo de energia utilizado neste trabalho é baseado em [15], que propõe um modelo empírico, obtido utilizando o hardware comercial TELOS [16]. Foi observado que, na Transmissão, o consumo de energia e o tamanho do pacote estão linearmente relacionados. Já para o estado de Recepção, verificou-se que o consumo é independente do tamanho do pacote. $\mathrm{O}$ mesmo ocorre para o estado Inativo. Neste trabalho, cada nó sensor pode estar associado a dois estados: Ativo ou Inativo. O estado Ativo possui ainda quatro sub-estados: Medição, Processamento, Transmissão e Recepção, o que pode ser visto na Figura 1. Já o sorvedouro, foi considerado com energia ilimitada, conectado a uma fonte externa. Portanto, pode-se classificar o modelo deste trabalho como híbrido, já que leva em conta o tamanho do pacote a ser transmitido, e ainda prevê a existência de estados, aos quais os nós podem estar associados.

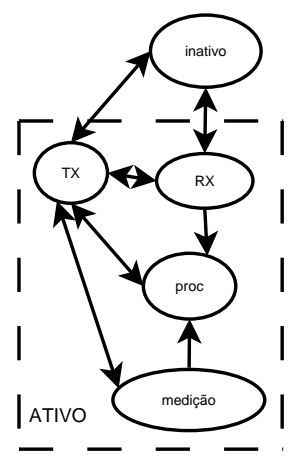

Fig. 1. Diagrama de estados dos nós.

Em [15], verificou-se que o consumo, na transmissão de 1 Byte, é de 0,034 mJ, com uma potência de transmissão de -5 $\mathrm{dBm}$. Observou-se também que essa transmissão leva cerca de $0,58 \mathrm{~ms}$. Já para os consumos na Recepção e no modo Inativo, assumindo um período de 1 segundo para cada um dos estados, foram obtidos $62,4 \mathrm{~mJ}$ e $1,8 \mathrm{~mJ}$, respectivamente. Neste trabalho incluiu-se o consumo fixo no modo Ativo de $10 \mathrm{~mJ}$, o que não foi utilizado em [15]. A inclusão deste consumo é válida, pois os nós podem estar Ativos, porém sem realizar tarefas, o que foi implementado no simulador, juntamente com o ajuste dos consumos dos demais estados do modelo de energia.

\section{Simulações}

Para o desenvolvimento das simulações, foi utilizado o TrueTime 1.5, um ambiente de simulação em tempo real baseado em MatLab / Simulink [17]. Foi utilizado o padrão IEEE 802.15.4 (Zigbee) nas simulações [18]. Os principais parâmetros fixos utilizados nas simulações estão apresentados na Tabela I.

A idéia é que cada nó sensor é fonte de informação, ou seja, mede amostras de um processo $f(x, y, t)$ e as envia para o destino. Foi utilizado, em todas as simulações, uma base de tempo de 0,1 segundos para esta tarefa. O processo $f(x, y, t)$ representa uma superfície variante no tempo, e seus valores dependem das coordenadas $x$ e $y$ dos nós e do tempo. Mais especificamente, o processo é descrito por:

$f(x, y, t)=\left[e^{\frac{-\left(x-m_{x}\right)^{2}}{2 \cdot d p_{x}^{2}}}+e^{\frac{-\left(y-m_{y}\right)^{2}}{2 \cdot d p_{y}^{2}}}\right] \times\left[e^{\frac{-\left(t-m_{t}\right)^{2}}{2 \cdot d p_{t}^{2}}}\right]+C$

Do processo em questão, $m_{x}=m_{y}=0$ e $m_{t}=40$, representam as respectivas médias de $x, y$ e $t ; d p_{x}=d p_{y}=$ 
TABELA I

PARÂmetros Fixos de SimulaçÃo.

\begin{tabular}{|c||c|}
\hline Energia Inicial dos Nós (J) & 2 \\
\hline Potência de Tx (dBm) & -5 \\
\hline Sensibilidade de Rx (dBm) & -66 \\
\hline Alcance do Rádio (m) & 10 \\
\hline Consumo no Modo Inativo (mJ/s) & 10 \\
\hline Consumo no Modo Ativo (mJ/s) & 18 \\
\hline Consumo no Modo Processamento (mJ/s) & 18 \\
\hline Consumo no Modo Rx (mJ/s) & 62,4 \\
\hline Consumo no Modo Tx (mJ/s) & 58,62 \\
\hline Tamanho do Pacote (Byte) & 1 \\
\hline
\end{tabular}

$d p_{t}=20$ são, respectivamente, os desvios padrões de $x, y$ e $t$, e $C=5$ é uma constante. Tal função foi utilizada, para que os algoritmos propostos sejam avaliados de maneira generalizada, já que não está sendo definida nenhuma aplicação específica. A Figura 2 ilustra a superfície descrita no instante de tempo $t=0$. Assim, para as coordenadas $x$ e $y$ de cada nó da rede, tem-se o valor medido, nesse instante.

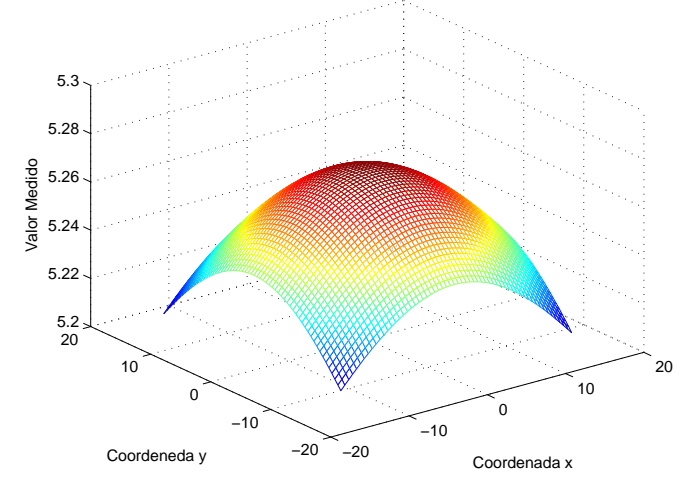

Fig. 2. Superfície monitorada em $t=0$.

As simulações foram realizadas utilizando comunicação de um salto, com o sorvedouro dentro do raio de alcance dos nós sensores. A cada rodada de simulação, o sorvedouro permanece fixo no centro da área monitorada, e os nós sensores são inseridos, ao seu redor, de forma aleatória. Uma aplicação que utiliza este tipo de comunicação é a chamada Rede de Sensores para o Corpo Humano, utilizada para monitoramento remoto de pacientes. Os sensores podem ser dispostos no corpo ou na roupa do paciente, de modo a coletar dados biométricos, e o sorvedouro, ou uma estação base, pode estar a poucos metros do paciente, por exemplo em um quarto do hospital, onde os dados podem ser coletados [19] [20].

\section{REsultados}

Esta Seção apresenta os resultados relativos às simulações dos algoritmos propostos. Em todos os casos, foram realizadas cinco simulações, e calculada a média dos resultados obtidos, com um intervalo de confiança de $95 \%$.

Inicialmente foram feitas simulações, onde cada nó, periodicamente, mede e transmite amostras diretamente ao sorvedouro, sem a utilização de nenhum tipo de economia de energia. Estes resultados foram utilizados como parâmetros de comparação com os algoritmos propostos. A Figura 3 apresenta o Tempo de Vida, definido quando a energia do primeiro nó sensor acaba, em função da quantidade de nós da rede. Foi utilizado o Tempo de Vida normalizado, em relação ao caso onde não se utilizou nenhum tipo de gerenciamento de energia.

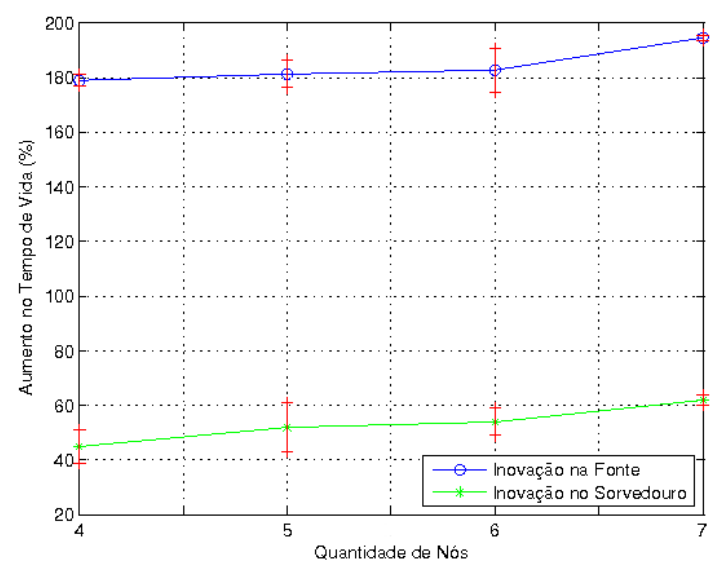

Fig. 3. Tempo de Vida normalizado x Escalabilidade.

A quantidade de nós utilizada nas simulações variou de quatro a sete. Em aplicações como Redes de Sensores para o Corpo Humano, pode-se utilizar poucos nós, já que estes podem ficar dispostos sobre o corpo ou na roupa dos pacientes. Logo, é justificável a utilização de uma rede com até sete nós, desde que haja a garantia de conectividade, através, por exemplo, da análise de métricas como a Taxa de Entrega de Pacotes [18]. Esta métrica foi analisada em todas as simulações deste trabalho, e os resultados obtidos foram superiores à $95 \%$, portanto satisfatórios.

Em relação à escalabilidade, aumentos entre $15 \%$ e $17 \%$ no Tempo de Vida da rede foram obtidos, o que pode ser visto na Figura 3. Segundo [21], com o aumento do número de nós sensores é possível aumentar o Tempo de Vida da rede, já que há um aumento do número de baterias em uso.

Porém, pode-se verificar um ganho máximo de $194 \%$ de aumento no Tempo de Vida, na abordagem baseada na fonte, onde verificou-se uma menor quantidade de transmissões, enquanto que a abordagem baseada no sorvedouro obteve $62 \%$ de aumento em relação às simulações sem a utilização dos algoritmos propostos.

Os próximos resultados apresentados foram obtidos através de simulações com uma quantidade fixa de sete nós.

A Figura 4 apresenta os resultados relativos ao Tempo de Vida da rede, em função do limiar de Inovação, em termos do Tempo de Vida normalizado. Os limiares de Inovação utilizados foram de $0,1 \%, 1 \%, 5 \%$ e $8 \%$.

Verifica-se, no algoritmo baseado na fonte, um aumento no Tempo de Vida da rede, com o aumento do limiar. Isto porque o aumento do limiar faz com que os nós apenas transmitam amostras medidas com variações percentuais cada vez maiores. Além disso, os nós permanecem inativos por tempos maiores, 


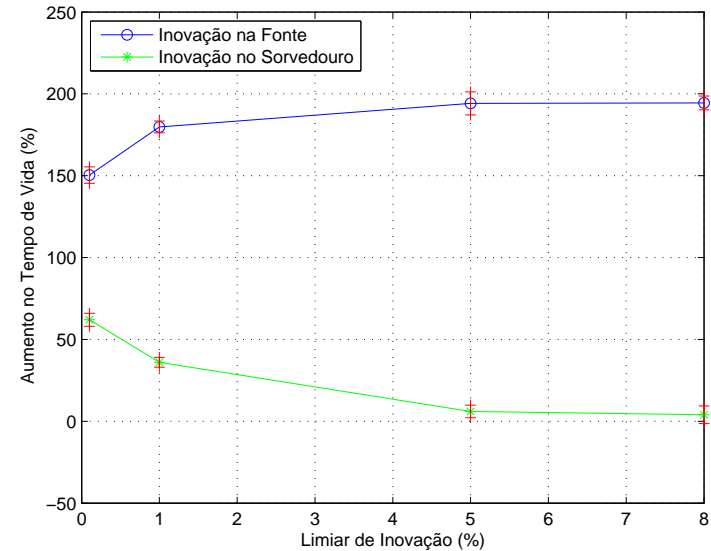

Fig. 4. Tempo de Vida normalizado x Limiar de Inovação.

entre as transmissões. Ou seja, a quantidade de transmissões é bastante reduzida, o que gera uma economia de energia maior.

Já no algoritmo baseado no sorvedouro, observa-se um comportamento diferente. Neste, os nós transmitem as amostras sem verificar se estas possuem, ou não, Inovação. A verificação da Inovação é realizada no próprio sorvedouro que, ao receber as amostras provenientes de cada nó sensor, decide, de forma centralizada, quando os nós ficarão Inativos e por quanto tempo. Portanto, quanto maior o limiar, mais mensagens o sorvedouro terá que receber para que se identifique a variação na medida e se decida os períodos de inatividade dos nós, já que o processo utilizado nos testes não possui variação rápida. Logo, não se reduz muito a quantidade de transmissões e, portanto, não se economiza tanto quanto no método baseado na fonte. Então, uma situação de limiar intermediário é interessante, pois não há necessidade de muitas transmissões para que o sorvedouro decida os períodos de inatividade dos nós. Ou seja, com um limiar grande, muitas transmissões e recepções podem ser necessárias para que haja Inovação e, portanto, pode não haver uma economia significativa de energia.

A Figura 5 apresenta o impacto do aumento do limiar de Inovação na redução percentual na quantidade de transmissões, para os dois algoritmos propostos, em relação às simulações sem a utilização do gerenciamento de energia.

Em relação ao algoritmo baseado na fonte, com o aumento do limiar, há uma redução significativa, de até $99,8 \%$, na quantidade de transmissões em relação às simulações sem o gerenciamento de energia. É importante lembrar que, com um limiar muito grande, a reduzida quantidade de transmissões pode ser insuficiente na reconstrução do processo, no sorvedouro. Logo, mesmo havendo uma economia grande de energia, o processo de comunicação pode vir a ser prejudicado. Isso remete, mais uma vez, à utilização de limiares intermediários, onde pode se conseguir uma economia de energia e, portanto, um aumento no Tempo de Vida da rede, e ainda não prejudicar a reconstrução do processo monitorado.

Já no método baseado no sorvedouro, observa-se um aumento na quantidade de transmissões. Com o aumento do limiar, o sorvedouro deve receber mais mensagens para que

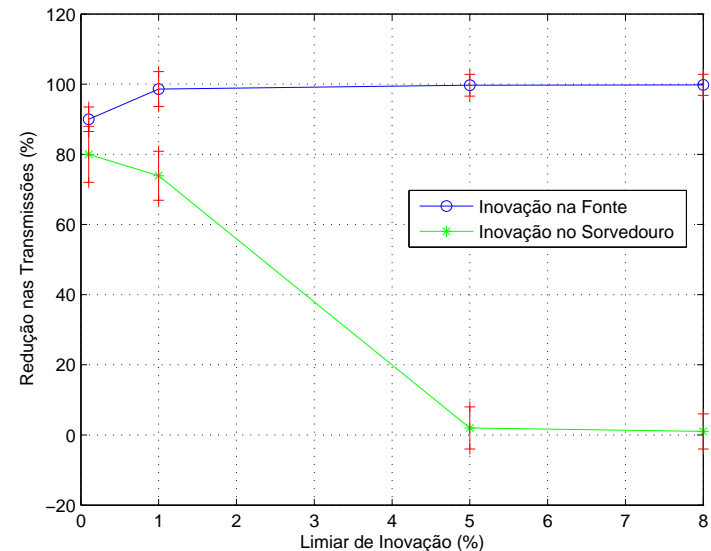

Fig. 5. Redução percentual nas transmissões x Limiar de Inovação.

se verifique o nível de variação de medida esperado.

Observa-se ainda, nas Figuras 4 e 5, que limiares a partir de $5 \%$ provocaram pequena variação, tanto no Tempo de Vida, quanto na redução de transmissões. Isto porque, para grandes valores de limiar, maiores variações entre medidas são necessárias para que haja Inovação.

A relação entre o aumento do limiar de Inovação e o erro na reconstrução do processo pode ser observada na Figura 6. No algoritmo baseado na fonte, verifica-se um aumento no erro máximo de reconstrução em função do aumento do limiar. Neste caso, o aumento do limiar provoca um decréscimo da quantidade de transmissões, o que impacta negativamente no erro, a partir do momento em que há menos amostras para se utilizar na reconstrução do processo. Já no sorvedouro, observa-se um decréscimo no erro máximo de reconstrução, com o aumento do limiar de Inovação. Ou seja, a diminuição da quantidade de transmissões e, portanto, da quantidade de informação disponível, leva a um aumento no erro máximo de reconstrução do processo.

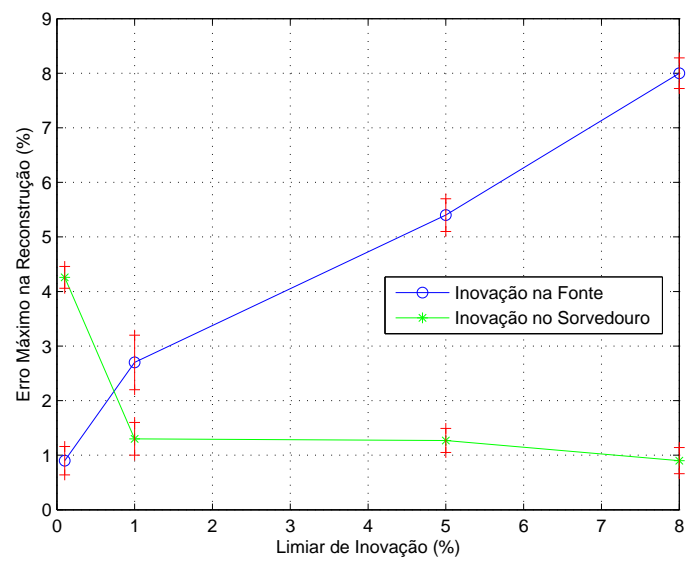

Fig. 6. Erro máximo na reconstrução do processo x Limiar de Inovação.

Neste trabalho, foram utilizadas medidas advindas de um processo genérico $f(x, y, t)$. Porém, se o objetivo fosse analisar 
temperatura, por exemplo, definir um limiar muito pequeno não seria interessante pois, com uma alta Taxa de Inovação, medidas com pouca variação seriam transmitidas desnecessariamente. Já em aplicações que necessitam de transmissões, mesmo de medidas com variações pequenas, a utilização de um limiar pequeno pode ser importante. Ou seja, o limiar e, portanto, o erro na reconstrução do processo, estão diretamente ligados à avaliação da aplicação específica, se for o caso. Desta forma, pode-se conjugar os três parâmetros: Tempo de Vida da rede, limiar de Inovação e erro na reconstrução, de modo a se conseguir uma RSSF mais autônoma.

\section{Conclusões e Trabalhos Futuros}

A partir dos resultados obtidos, verifica-se que os algoritmos propostos se mostraram úteis, já que conseguiu-se reduções significativas na quantidade de transmissões e, por conseguinte, um aumento na sobrevida da rede. A utilização da inatividade dos nós também se mostrou importante, já que se torna possível economizar energia em períodos que não estão transmitindo. Ou seja, economiza-se com a diminuição das transmissões e, também, com a inatividade dos nós.

Além disso, a avaliação do compromisso entre Tempo de Vida e erro na reconstrução do processo é importante, o que vem de encontro à aplicação que se deseja avaliar. Com a utilização de um limiar alto, a redução das transmissões pode impactar de forma negativa a reconstrução do processo monitorado, o que foi mais latente no algoritmo baseado na fonte, já que os nós apenas transmitem, caso as medidas sejam maiores do que o limiar. Já em relação ao método baseado no sorvedouro, limiares altos representam que este deve receber muitas mensagens, para que se consiga uma amostra com uma variação desejada e, com isso, além de haver muitas transmissões/recepções na rede, os nós demoram muito para entrar no modo de economia de energia. A utilização de limiares intermediários pode ser a solução para estas questões.

Pretende-se, para os próximos trabalhos, verificar algumas questões, como o impacto do aumento da quantidade de nós e o uso de roteamento em relação aos resultados já obtidos. Também pretende-se analisar a utilização de um tempo de inatividade inicial. Isto pode ser útil, principalmente no algoritmo baseado no sorvedouro, em situações onde ele demora muito para encontrar medidas com Inovação. Além disso, desejase verificar o impacto da energia inicial dos nós, já que, no começo, estes gastam energia na realização de medidas e processamento até encontrarem uma amostra com Inovação e, com isso, poderem dormir. Por fim, pretende-se verificar a utilização de outros métodos de estimação para os tempos de inatividade, para que os nós possam permanecer Inativos por períodos maiores, entre transmissões com Inovação.

\section{REFERÊNCIAS}

[1] W. M. Aioffi e G. R. Mateus, "Otimização do Controle de Densidade com Sorvedouros Móveis em Redes de Sensores”, Simpósio Brasileiro de Redes de Computadores, pp. 323-336, 2007.

[2] A. A. F. Loureiro, "Redes de Sensores Sem Fio", Simpósio Brasileiro de Redes de Computadores, pp. 179-226, 2003.

[3] I. F. Akyildiz, W. Su, Y. Sankarasubramaniam e E. Cayirci., "Wireless sensor networks: A survey", Computer Networks, v. 38, pp. 393-422, 2002.
[4] S. Kostin e C. L. Amorim, "Controle de Potência de Transmissão para Redes de Sensores em Cenários com Obstáculos", Simpósio Brasileiro de Redes de Computadores, pp. 337-350, 2007.

[5] I. Teixeira, J. F. Resende e A. C. P. Pedroza, "Wireless Sensor Networks: Improving the Network Energy Consumption", Simpósio Brasileiro de Telecomunicações, 2004

[6] C. Intanagonwiwat, R. Govindan e D. Estrin, "Directed Diffusion: A Scalable and Robust Communication Paradigm for Sensor Networks", Proceedings of ACM MobiCom'00, pp. 56-67, 2000.

[7] R. B. Araújo, L. A. Villas e A. Boukerche, "Uma Solução de QoS com Processamento Centrado para Redes de Atuadores e Sensores sem Fio", Simpósio Brasileiro de Redes de Computadores, pp. 309-322, 2007.

[8] D. O. Cunha, R. P. Laufer, I. M. Moraes, M. D. D. Bicudo, P. D. Velloso e O. C. M. B. Duarte, "Bio-Inpired Field Estimation With Wireless Sensor Networks", 12th International Conference on Telecommunications - ICT'2005, 2005

[9] I. F. Akyildiz, M. C. Vuran e O. B. Akan, "On Exploiting Spatial and Temporal Correlation in Wireless Sensor Networks", in Proc. WiOpt'04: Modeling and Optimization in Mobile, Ad Hoc and Wireless Networks, 2004.

[10] A. J. G. Pinto, J. Stochero e J. F. Rezende, "Descoberta de Rotas e Mecanismo de Agragação em Redes de Sensores", Simpósio Brasileiro de Redes de Computadores, pp. 567-570, 2004.

[11] M. Vetterli, P. Marziliano e T. Blu, "Sampling Signals With Finite Rate of Innovation", IEEE Transactions on Signal Processing, v. 50, pp. 1417-1428, 2002.

[12] Y. Sung, L. Tong e A. Ephremides, "Route Selection for Detection of Correlated Random Fields in Large Sensor Neworks", Conference on Information Sciences and Systems, pp. 1-6, 2005.

[13] P. S. Sausen, J. R. B. Sousa, M. A. Spohn, A. Perkisich e A. M. N. Lima, "Dynamic Power Management With Scheduled Switching Modes in Wireless Sensor Networks", Proceedings of the 15th Annual Meeting of the IEEE International Symposium on Modeling, Analysis, and Simulation of Computer and Telecommunication Systems (MASCOTS'07, pp. 1-9, 2007.

[14] D. O. Cunha, "Conservação de Energia em Redes Ad Hoc", Dissertação de Mestrado, Universidade Federal do Rio de Janeiro, COPPE, 2004.

[15] Y. Panthachai e P. Keeratiwintakorn, "An Energy Model for Transmission in Telos-based Wireless Sensor Networks", Proceedings of the 4th International Joint Conference on Computer Science and Software Engineering, 2007.

[16] J. Polastre, R. Szewczyk e D. Culler, "Telos: Enabling Ultra-Low Power Wireless Research", Proceedings of the 4th International Symposium on Information Processing in Sensor Networks, 2005.

[17] K.-E. Arzén, M. Ohlin, A. Cervin, P. Alriksson e D. Henriksson, "Holistic Simulation of Mobile Robot and Sensor Networks Applications Using TrueTime", Proceedings of the European Control Conference, pp. 4301-4308, 2007.

[18] J. Zheng e M. J. Lee, "A Comprehensive Performance Study of IEEE 802.15.4", IEEE Press Book, 2004.

[19] P. S. Pandian, K. P. Safeer, P. Gupta, D. T. Shakunthala, B. S. Sundersheshu e V. C. Padaki, "Wireless Sensor Network for Wearable Physiological Monitoring”, Journal of Networks, Vol. 3, No. 5, pp. 21-29, 2008.

[20] G. Virone, A. Wood, L. Selavo, Q. Cao, L. Fang, T. Doan, Z. He, R. Stoleru, S. Lin e J. A. Stankovic, "An Advanced Wireless Sensor Network for Health Monitoring", Proceedings of the First Distributed Diagnosis and Home Healthcare Conference, pp. 95-100, 2006.

[21] I. G. Sene Jr, T. M. G. A. Barbosa, A. F. Rocha, F. A. O. Nascimento e H. S. Carvalho, "Monitoração de Temperatura Corporal Baseada em uma Rede de Sensores Sem Fios", XVIII Congresso Brasileiro de Informática em Saúde (CBIS), 2006. 